\title{
LOS ORGANISMOS PARTICIPATIVOS PARA POLÍTICAS PÚBLICAS DE LA AGRICULTURA FAMILIAR, CAMPESINA E INDÍGENA
}

\section{The Participatory Bodies for Public Policies on Family, Peasant and Indigenous Farming}

Fecha recepción: 21 de septiembre de 2020 / fecha aceptación: 11 de enero de 2021

Rodrigo Palleres Balboa ${ }^{1}$ Edgardo Gabriel González ${ }^{2}$ y Guillermo Miguel Hang ${ }^{3}$

Cómo citar este artículo:

Palleres Balboa R., González E. y Hang G. (2021). Los organismos participativos para políticas públicas de la agricultura familiar, campesina e indígena. Revista Pensamiento y Acción Interdisciplinaria, 7(1), 129-145. http://doi.org/10.29035/pai.7.1.129

\begin{abstract}
Resumen
La agricultura familiar en Argentina ha recorrido un largo proceso de institucionalización y constitución como actante político. El 15 de agosto de 2014 mediante la Resolución 571/2014 del Ministerio de Agricultura, Ganadería y Pesca se creó el Consejo de la Agricultura Familiar, Campesina e Indígena. En los considerandos de dicha resolución se menciona la necesidad de contar con un espacio al interior del ministerio "de articulación institucional que agrupe a las unidades de gestión y a las Organizaciones Campesinas, Indígenas y de la Agricultura Familiar de alcance nacional". Mediante una entrevista estructurada a los y las representantes de las organizaciones de la agricultura familiar, se buscará describir y comprender cuál es la percepción que tienen estas personas que integran el Consejo acerca del mismo, en tanto, herramienta participativa para la toma de decisiones de políticas públicas. A partir de las respuestas proporcionadas se realizará un contraste entre ellas, los diferentes grados de participación y los objetivos que la normativa que crea el Consejo y la ley 27.118 de agricultura familiar, campesina e indígena establecen para el mismo.
\end{abstract}

Palabras clave: Agricultura familiar, Democracia, Gobernanza, Participación, Políticas públicas

1 Abogado, Universidad Nacional de La Plata, Buenos Aires, Argentina. Correo electrónico: rodrigo_palleres@hotmail.com 2 Abogado, Universidad Nacional de La Plata, Buenos Aires, Argentina. Instituto Nacional de Tecnología Agropecuaria INTA. Correo electrónico: abogadoegonzalez@yahoo.com.ar

3 Facultad de Ciencias Agrarias y Forestales. Departamento de Desarrollo Rural. Universidad Nacional de La Plata, Buenos Aires, Argentina. Correo electrónico: ecagraria@agro.unlp.edu.ar 


\begin{abstract}
Family farming in Argentina has gone through a long process of institutionalization and constitution as a political actor. On August 15, 2014, through Resolution 571/2014 of the Ministry of Agriculture, Livestock and Fisheries, the Council for Family, Peasant and Indigenous Farming was created. The recitals of said resolution mention the need to have a space within the ministry "of institutional articulation that groups together the management units and the Peasant, Indigenous and Family Farming Organizations of national scope". Through a structured interview with the representatives of the family farming organizations, it will be sought to describe and understand what the perception that these people who make up the Council have about it as a participatory tool for making public policy decisions. Based on the answers provided, a contrast will be made between them, the different degrees of participation and the objectives that the normative that creates the Council and Law 27.118 on family, peasant and indigenous farming establish for it.
\end{abstract}

Keywords: Family farming, Democracy, Governance, Participation, Public policies

\title{
Introducción
}

La agricultura familiar en Argentina ha recorrido un largo proceso de institucionalización y constitución como actante político. En la literatura sobre el tema se considera como uno de los momentos iniciales la creación de la Reunión Especializada sobre Agricultura Familiar en el año 2004 en el marco del MERCOSUR mediante la Resolución del $\mathrm{GMC}^{4}$ 11/04. Este espacio de discusión sobre problemáticas comunes a los países miembros fue creado a instancias de Brasil con el objetivo de fortalecer las políticas públicas para el sector, promover el comercio de los productos de la agricultura familiar y facilitar la comercialización en la región. ${ }^{5}$

En Argentina se produce un movimiento reflejo, por el cual se crea en 2004 la Comisión de Agricultura Familiar en el marco del Ministerio de Relaciones Exteriores y Comercio Exterior promovida por la Federación Agraria Argentina (FAA). Esta organización, que nuclea en el país a los pequeños y medianos productores, participó activamente desde entonces en la construcción de un espacio de discusión y representación política: el Foro Nacional de la Agricultura Familiar (FONAF). Este foro buscó congregar a todas las organizaciones de la agricultura familiar en el país, con el objetivo de presentar propuestas de políticas públicas agrarias. El trabajo conjunto de las organizaciones, en encuentros que duraron varias jornadas, desembocó en la redacción y presentación de dos documentos: el Documento Elaborado por las Organizaciones Representativas del Sector Productor Agropecuario Familiar de 2006 (también conocido como Documento Base Mendoza 2006) y las Propuestas para un Plan Estratégico de Desarrollo Rural

\footnotetext{
4 Abreviatura de Grupo Mercado Común.

5 Extraído de la página oficial http://www.reafmercosul.org/sobre-reaf último acceso 11/9/2020
} 
de 2008 (también denominado Documento Base 2008). Su funcionamiento se vio reconocido por el Estado el 29 de marzo de 2006 mediante la Resolución 132/2006 de la Secretaría de Agricultura, Ganadería, Pesca y Alimentación y nuevamente el 12 de enero de 2011 mediante la Resolución 8/2011 del Ministerio de Agricultura, Ganadería y Pesca.

A la par que se producía este proceso de movilización de actantes el Estado acompañó con otra decisión clave de política pública: la creación del Centro de Investigación y Desarrollo Tecnológico para la Agricultura Familiar (CIPAF) en la órbita del Instituto Nacional de Tecnología Agropecuaria (INTA) el 26 de agosto de 2005. El CIPAF tiene líneas nacionales de investigación relacionadas con las necesidades de la agricultura familiar (agua, agroecología, caracterización de los actores, comercialización, maquinarias y herramientas, energías renovables, recursos genéticos, tierra y bienes naturales) y se estructura a partir de cinco Institutos de Investigación y Desarrollo Tecnológico para la Agricultura Familiar (IPAF), cada uno en una de las cinco macrorregiones de Argentina: Región Noroeste (NOA), Región Noreste (NEA), Región Pampeana, Región Cuyo y Región Patagonia.

Otra política pública dirigida a los y las integrantes de la agricultura familiar, y que significó un paso más en el proceso de institucionalización de la misma, fue la ley 26.117 de "promoción del microcrédito" sancionada el 28 de junio de 2006. Esta ley creó la Comisión Nacional de Promoción del Microcrédito para el Desarrollo de la Economía Social (CONAMI) en el marco del entonces Ministerio de Desarrollo Social de la Nación. Esta herramienta "ha sido muy útil para facilitar la compra de insumos, que han permitido en muchos casos aumentar los volúmenes de producción, especialmente en aquellos productores más pequeños insertos en canales de comercialización efectivos" (Gonzalez, et al., 2013, p. 35).

El 23 de octubre de 2007 mediante la Resolución 255/2007 de la Secretaría de Agricultura, Ganadería, Pesca y Alimentos se creó el Registro Nacional de Agricultura Familiar con el objetivo de identificar a los agricultores familiares, a través de un registro que califique y habilite a los inscriptos para acceder a las políticas públicas que les estén específicamente destinadas ${ }^{6}$. Este instrumento permitió aumentar la visibilidad de los y las integrantes de la agricultura familiar en el campo de disputa de políticas públicas.

El 28 de febrero de 2008 se sancionó otra política para la agricultura familiar: la ley 26.355 de marcas colectivas. Esta ley crea un "signo distintivo que identifique a los productos y servicios elaborados/prestados por emprendimientos de la economía social, representando los valores del trabajo asociativo, el precio justo y la identidad local" (Gonzalez, et al.2013, p. 35). Con el Decreto 571/2008 del 4 de abril de 2008 se creó en el marco del entonces Ministerio de Economía y Producción la Subsecretaría de Desarrollo Rural y Agricultura Familiar dependiente de la Secretaría de Agricultura, Ganadería, Pesca y Alimentos. Desde

6 Extraído de los considerandos de la resolución. Puede encontrarse en shorturl.at/hBL05 último acceso 11/9/2020. 
allí se impulsó la creación del Monotributo Social Agropecuario en el año 2009. Esta herramienta permitía a los y las productoras emitir facturas, poder realizar contratos de suministro con el Estado, acceder a una obra social y poder realizar aportes jubilatorios.

También en 2009, el primero de octubre, se creó la Comisión de la Agricultura Familiar (SENAF) en el ámbito del Servicio Nacional de Sanidad y Calidad Agroalimentaria (SENASA) ${ }^{7}$ mediante la Resolución 759/2009. Esta comisión tiene como objetivo construir, participativamente, nuevas normas que regulen la producción de alimentos, y adecuar las vigentes contemplando las características propias del sector ${ }^{8}$.

En el año 2012 el CIPAF impulsó la creación del Foro Nacional de Universidades para la Agricultura Familiar (Foro UNAF). Este foro "está compuesto por representantes de las Universidades Nacionales de las cinco regiones del país: NOA, NEA, Pampeana, Cuyo y Patagonia, bajo la coordinación de los IPAF regionales" (Fernández, 2018, p. 231) y tiene como principal objetivo generar tecnologías y procesos sociales más participativos y democráticos dentro de una estructura multiactoral (Hang, et al., 2015).

El 15 de agosto de 2014 mediante la Resolución 571/2014 del Ministerio de Agricultura, Ganadería y Pesca se creó el Consejo de la Agricultura Familiar, Campesina e Indígena. En los considerandos de dicha resolución se menciona la necesidad de contar con un espacio al interior del ministerio de articulación institucional, de alcance nacional, que agrupe a las unidades de gestión y a las Organizaciones Campesinas, Indígenas y de la Agricultura Familiar ${ }^{9}$. También se hace alusión a la posibilidad de invitar como miembros no permanentes a representantes de otros ministerios y/u organismos públicos nacionales (en tanto sea pertinente para la implementación de programas en particular) y se explicita que el Consejo es un ámbito participativo para debatir y generar consensos sobre diseños e implementación de políticas públicas estratégicas ${ }^{10}$.

El 17 de diciembre de 2014 se sancionó la ley 27.118 de "Reparación histórica de la agricultura familiar para la construcción de una nueva ruralidad en la Argentina", también conocida como "Ley de agricultura familiar, campesina e indígena". Esta ley en su artículo 9 referencia expresamente al Consejo de la Agricultura Familiar, Campesina e Indígena como uno de los participantes junto con la autoridad de aplicación de la ley en la ejecución de la misma. Sobre el funcionamiento y la percepción de los integrantes de este espacio de articulación se tratarán los apartados siguientes.

\footnotetext{
7 El SENASA es el "encargado de ejecutar las políticas nacionales en materia de sanidad y calidad animal y vegetal e inocuidad de los alimentos de su competencia, así como de verificar el cumplimiento de la normativa vigente en la materia". Fragmento extraído de https://www.argentina.gob.ar/senasa/que-es Último acceso el 11/9/2020.

8 Extraído de la página web oficial de la SENAF shorturl.at/fpwxL Último acceso el 11/9/2020.

9 Extraído de shorturl.at/sxLY9 Último acceso el 11/9/2020.

10 Ibídem.
} 
Partiendo desde un enfoque de redes de políticas públicas basado en el institucionalismo centrado en los actores (Scharpf, 1997) este trabajo se propone indagar acerca de las percepciones y preferencias de los y las representantes de las organizaciones de la agricultura familiar que integran el Consejo de la Agricultura Familiar, Campesina e Indígena. La construcción de esta información es relevante en la medida que, siguiendo este enfoque, las percepciones y preferencias de los actores tienen una relación dialéctica con las características de las estructuras institucionales en las que desarrollan su actividad relacionada con las distintas fases de las políticas públicas. Específicamente se indagará acerca de las mejoras o beneficios que significó para la organización participar en el Consejo, las mejoras o beneficios percibidos para la agricultura familiar a partir de la existencia del Consejo y sobre la valoración del funcionamiento del espacio.

\section{Marco referencial}

Vivimos en sociedades de democracias de baja intensidad (De Sousa Santos, 2006). La democracia es uno de los instrumentos hegemónicos que integran las condiciones de convivencia legítima a nivel político y social. En los últimos 30 años, a raíz de la crisis del modelo del Estado como solución a las problemáticas surgidas en la sociedad civil, ha ocurrido una pérdida de "demo-diversidad" consistente en la desaparición de las alternativas contra-hegemónicas a la democracia representativa liberal (la democracia popular, la democracia desarrollada en países a partir del colonialismo y particularmente la democracia participativa). Esta crisis se caracteriza además por la desaparición de la tensión entre capitalismo y democracia (en tanto esta última no se constituye en una idea-fuerza de redistribución social), la inversión de la conceptualización del Estado como solución a la del Estado como problema (para el que la sociedad civil sería la solución en tanto el Estado es "ineficiente") y la concepción de la necesidad de un Estado débil para que exista una sociedad civil fuerte (en vez de un reflejo en el que un Estado fuerte democráticamente resulte en una sociedad civil fuerte). A esto se suma el surgimiento de un constitucionalismo global de las empresas multinacionales (De Sousa Santos, 2006) por el cuál las leyes nacionales se desjerarquizan y dejan de tener prioridad ante los acuerdos impuestos por estos actores mundiales.

En este contexto de puesta en cuestionamiento del horizonte de una democracia participativa debemos precisar lo que entendemos por participación. Siguiendo las ideas descriptas por Frans Geilfus podemos decir que la participación no es un estado fijo, sino un proceso mediante el cual la gente puede ganar más o menos grados de participación en el proceso de desarrollo (Geilfus, 2002). Para describir los diferentes grados de participación propone siete niveles:

1. Pasividad: la participación está limitada a recibir información y no hay ninguna incidencia en las decisiones o la implementación de lo planificado. 
2. Suministro de información: la participación se limita a responder encuestas sin que exista posibilidad de influir en el uso que se le dará a esa información.

3. Participación por consulta: la participación consiste en la consulta por parte de agentes externos que escuchan el punto de vista proveído sin que exista posibilidad de incidir en las decisiones que se tomarán a raíz de dichas consultas.

4. Participación por incentivos: la participación se materializa mediante trabajo u otros recursos que se otorgan a cambio de incentivos (materiales, sociales, capacitación) sin que haya ninguna incidencia directa en las decisiones de lo planificado.

5. Participación funcional: la participación se realiza mediante la conformación de grupos de trabajo que responden a objetivos predeterminados. No hay incidencia sobre la formulación de los objetivos, pero el desarrollo de las actividades es monitoreado y se realizan los ajustes necesarios.

6. Participación interactiva: la participación recae en la formulación, implementación y evaluación de los proyectos, con procesos de enseñanza-aprendizaje y la toma de control en forma progresiva del proyecto.

7. Auto-desarrollo: la participación se materializa en la toma de iniciativas sin necesidad de una intervención externa.

Como se vio en la introducción la agricultura familiar, a partir del proceso de institucionalización a nivel nacional y regional, fue consiguiendo mayores grados de participación en las políticas públicas, esto visto a partir tanto de la creación de estructuras organizativas al interior del Estado específicas para sus integrantes como por la creación de políticas públicas específicas. Habida cuenta de que la categoría de agricultura familiar está en permanente construcción (Schiavoni, 2010) nos interesa movilizar sucintamente dos conceptualizaciones que consideramos relevante. Se trata, en primer lugar, de la propuesta en el mencionado Documento Base Mendoza 2006 en la que se puede leer:

En nuestro concepto, la agricultura familiar es una "forma de vida" y "una cuestión cultural", que tiene como principal objetivo la "reproducción social de la familia en condiciones dignas", donde la gestión de la unidad productiva y las inversiones en ella realizadas es hecha por individuos que mantienen entre sí lazos de familia, la mayor parte del trabajo es aportada por los miembros de la familia, la propiedad de los medios de producción (aunque no siempre de la tierra) pertenece a la familia, y es en 
su interior que se realiza la transmisión de valores, prácticas y experiencias (Foro Nacional de la Agricultura Familiar, 2006).

Y en segundo lugar la definición que se encuentra en el documento base, redactado por el CIPAF, del "Programa Nacional de Investigación y Desarrollo Tecnológico para la Pequeña Agricultura Familiar (PNPAF)":

Se entiende a la agricultura familiar como un tipo de producción donde la unidad doméstica y la unidad productiva están físicamente integradas, la agricultura es un recurso significativo en la estrategia de vida de la familia, la cual aporta la fracción predominante de la fuerza de trabajo utilizada en la explotación, y la producción se dirige tanto al autoconsumo como al mercado (INTA, 2005).

La agricultura familiar así entendida en el marco del proceso de institucionalización pasó además por un proceso de creciente asociativismo que no fue espontáneo "sino que se encuentra fuertemente atado a las políticas públicas para la agricultura familiar (donde conformar grupos de productores aparece como una exigencia para la participación en proyectos o la obtención de beneficios)" (Ambort, 2017, p. 10). Por otra parte la organización al interior de la agricultura familiar es una de "las estrategias que las familias productoras han logrado articular para sostener sus actividades y modos de vida, en un contexto altamente desfavorable y exigente en términos de adecuación a la competitividad de los más capitalizados" (Ambort, 2017, p. 20).

En este contexto se crea el Consejo de la Agricultura Familiar, Campesina e Indígena por el Ministerio de Agricultura, Ganadería y Pesca en al año 2014. La Resolución 571/2014 por la cual se lo constituye establece en su artículo dos la integración del Consejo:

- Las máximas autoridades (en calidad de titulares y en calidad de suplentes los funcionarios que estas autoridades determinen) de:

- La Secretaría de Agricultura Familiar del Ministerio de Agricultura, Ganadería y Pesca

- El Instituto Nacional de Tecnología Agropecuaria

- El Servicio Nacional de Sanidad y Calidad Agroalimentaria

- La Unidad para el Cambio Rural

- Un representante de cada organización Campesina, Indígena y de la Agricultura Familiar de alcance nacional. 
Se debe destacar que en la norma de creación (ni en ninguna otra) se establece taxativamente cuáles serán las organizaciones de alcance nacional que integrarán el Consejo. Esta ausencia de definición le da al Consejo un carácter dinámico en tanto la presencia de las organizaciones en el mismo es tanto un reconocimiento por parte del Estado como por parte del resto de las organizaciones que integran el Consejo (como se resalta en el apartado de resultados y discusión).

\section{Metodología}

Este trabajo se realizará con una perspectiva no estándar (Marradi, et al., 2007) y utilizando técnicas cualitativas de recolección de datos. Mediante una entrevista estructurada en la que se realizarán de forma telemática tres preguntas a los y las representantes de las organizaciones de la agricultura familiar se buscará, siguiendo la tradición hermenéutica del enfoque no estándar de las ciencias sociales, describir y comprender cuál es la percepción que tienen estas personas que integran el Consejo acerca del mismo en tanto herramienta participativa para la toma de decisiones de políticas públicas.

Las preguntas que se consideraron relevantes para la entrevista son las siguientes:

1. ¿Qué beneficios entiende que significó para su organización la participación en el Consejo?

2. ¿Qué beneficios entiende que significó para la agricultura familiar la existencia del Consejo?

3. ¿Cómo valora la instancia de funcionamiento del Consejo?

A partir de las respuestas proporcionadas se realizará un contraste entre ellas, los diferentes grados de participación y los objetivos que la resolución normativa que crea el Consejo y la ley 27.118 establecen para el mismo en el apartado de resultados y discusión.

El Consejo está integrado actualmente por diecisiete organizaciones de la agricultura familiar, campesina e indígena ${ }^{11}$. Si bien esta investigación tiene un carácter exploratorio, se entrevistará a la mayor cantidad posible de representantes (teniendo como parámetro inicial conseguir la respuesta de al menos un o una representante por organización), con el conocimiento de que no se busca llegar a conclusiones representativas sino más bien a comprender "los procesos a través de los cuáles los sujetos producen interpretaciones que dan forma a lo real en la vida cotidiana" (Marradi, et al., 2007, p. 40), lo que en este caso recae en el funcionamiento del espacio del que forman parte. 


\section{Resultados y discusión}

Para comenzar a contrastar y organizar la información recolectada mediante las entrevistas, se expresarán los objetivos declarados del Consejo de la Agricultura Familiar, Campesina, Indígena en su normativa de creación la Resolución 571/2014 del Ministerio de Agricultura, Ganadería y Pesca de la Nación. La misma en su primer artículo establece que el Consejo es un espacio participativo para debatir y generar consensos sobre diseños e implementación de políticas públicas estratégicas para el desarrollo sustentable y consolidación de la Agricultura Familiar, Campesina e Indígena.

Además cabe mencionar algunos fundamentos de los considerandos de la resolución en los cuales se señala que el Consejo se crea como un espacio de articulación institucional que agrupe a las unidades de gestión y a las Organizaciones Campesinas, Indígenas y de la Agricultura Familiar de alcance nacional, y nace como un ámbito participativo para debatir y generar consensos sobre diseños e implementación de políticas públicas estratégicas para el desarrollo, la producción de alimentos y el arraigo rural, jerarquizando las políticas públicas hacia la Agricultura Familiar, y que su creación generará posibilidades de potenciar los resultados que se esperan de los múltiples programas en curso, para lograr un mayor y eficaz aprovechamiento de los recursos financieros, técnicos y administrativos disponibles para el sostenimiento de la Agricultura Familiar, Campesina e Indígena.

Por su parte la ley 27.118 de agricultura familiar, campesina e indígena, se refiere al Consejo en su artículo nueve en el que establece que el Ministerio de Agricultura, Ganadería y Pesca es la autoridad de aplicación de la ley y que da participación al Consejo de Agricultura Familiar, Campesino, Indígena, creado por resolución 571 de MAGyP.

Teniendo en cuenta estas caracterizaciones realizadas por los instrumentos normativos que operativizan el espacio de articulación se puede considerar, teniendo en cuenta el marco referencial planteado, que el Consejo se estructura como un espacio de participación de nivel tres, es decir, donde las organizaciones participan aportando su punto de vista, sin que exista una disposición normativa que les permita incidir en las decisiones que se toman sobre los temas que se someten a discusión.

A partir de las respuestas de los y las representantes de las organizaciones que conforman el Consejo se ve que el objetivo de constituirse como un espacio o ámbito participativo para el debate y la generación de consensos de políticas públicas se ve alcanzado de diferentes formas. Se valora positivamente la existencia de una "instancia de diálogo entre el Estado y las organizaciones de agricultores" (ACINA) en la que se pueda conseguir información valiosa para las organizaciones como "todos los programas que hay en los distintos ministerios" (FNC) y en donde se pueden "desde los territorios expresar demandas, necesidades y propuestas" 
(MNCl-Somos Tierra) para lograr que el Estado tenga un "ida y vuelta con las organizaciones" (CANPO), que recepte las "sugerencias y recomendaciones de políticas públicas" (Frente Agrario Evita) e "intercambiar ideas y acciones que se puedan implementar" (UAPA).

Dos procesos en particular fueron resaltados en varias entrevistas: el de sanción de la ley 27.118 en el año 2014 y el de la redacción de su reglamentación (que continúa en proceso). A este respecto se señaló como una instancia valiosa el hecho de haber podido participar desde el Consejo en la redacción de la ley "donde pudimos canalizar la mayoría de nuestras inquietudes" (AGA). Además se señala al Consejo como un lugar de "impulso y empuje para la reglamentación de la ley de agricultura familiar" (MCL) y donde existe la posibilidad para las organizaciones de "discutir la reglamentación de la ley" (FECOFE).

En cuanto a la finalidad declarada del Consejo de ser un espacio de articulación institucional entre las unidades de gestión y las organizaciones campesinas, indígenas y de la agricultura familiar de alcance nacional surgió en las entrevistas la importancia asignada por los y las representantes al Consejo como un lugar de encuentro, además de para dialogar con el Estado, para articular entre las propias organizaciones. Esto apareció relacionado a los beneficios que significó el Consejo para la organización: "a nosotros como organización principalmente nos sirvió para conocer y articular algunas cosas con respecto a otras organizaciones" (FNC) y también como oportunidad "de vincularnos con una cantidad de organizaciones de todo el país" (FECOFE).

Este mismo aspecto de valorización de la posibilidad de articulación entre las organizaciones también se vio resaltado en relación al funcionamiento del Consejo desde su creación en 2014 hasta las elecciones presidenciales de 2015 en las cuáles se produjo un cambio en el frente político a cargo del Poder Ejecutivo Nacional: "nosotros veíamos con muy buenos ojos cuando esos encuentros duraban dos días, que nos permitía el primer día interiorizarnos en qué era lo que estaba pasando en cada una de las organizaciones, nos daba la oportunidad de consensuar la agenda que íbamos a tener con el Estado" (ACINA). En el mismo sentido se valora como positiva la existencia del Consejo en tanto "nos posibilitó intercambiar experiencias, poder poner puntos en común con las demás organizaciones con desarrollo también nacional, entender que las problemáticas, los desafíos y los enfoques son los mismos, y que nos fortalece la unidad, la organización y la participación" (Frente Agrario Evita).

Otro elemento que surge de las entrevistas relacionado a los beneficios que significa la existencia del Consejo se relaciona con el reconocimiento y la valorización tanto de cada organización que forma parte del mismo, como de la diversidad territorial que representan al ser de alcance nacional y de la agricultura familiar, campesina e indígena en su conjunto. 
Respecto de la importancia para las organizaciones de formar parte del Consejo se resaltó que "el valor político importante fue que se las reconociera como organizaciones agrarias representativas" (ACINA), el hecho de haber otorgado "visibilidad y presencia en el debate nacional junto a otras organizaciones" (AGA) y la "visibilización de las mujeres rurales y la posibilidad de discutir las políticas públicas" (AMRAF).

En cuanto a la presencia de organizaciones de alcance nacional se rescató el carácter representativo del Consejo "de toda la diversidad de la agricultura familiar a lo largo y ancho de Argentina" (Frente Agrario Evita), la posibilidad del Consejo de dar "una mirada mucho más federal, mucho más amplia" (CANPO) sobre los debates y consensos a construir y su importancia en tanto su participación contribuye en "ampliar y diversificar todo lo que tiene que ver con la constitución y la conducción de lo agrario en Argentina" (ACINA).

Finalmente y con mucha presencia en las entrevistas realizadas se presenta como un beneficio de la existencia del Consejo la valorización de la agricultura familiar, campesina e indígena. Así se señaló que uno de los motivos fundamentales de creación del Consejo fue "que la agricultura familiar, a través de sus organizaciones, por principio empezara a ser un tema de debate" (ACINA). En el mismo sentido se expresó que la creación del "Consejo de la Agricultura Familiar fue una larga lucha donde muchas organizaciones veníamos planteando la necesidad de ser protagonistas también en las discusiones, en la planificación de la política pública para el sector, incluso en la ejecución de la política pública" ( $\mathrm{MNCl}$-Somos Tierra) y que "para la agricultura familiar fue empoderarla el hecho de que varias organizaciones estemos en el Consejo de la Agricultura Familiar, proponiendo, compartiendo temas importantes" (AGA).

Ya no vinculados a los objetivos declarados por la normativa que crea el Consejo pero igualmente referidos a su funcionamiento y finalidad surgieron dos elementos más en las entrevistas: la posibilidad de "mejorar" el Consejo y un rol del Consejo "más allá" de sus funciones asignadas.

Respecto de las posibles mejoras que podrían ocurrir en el funcionamiento del Consejo se resaltó su rol como "consejo asesor": "sabemos que es un consejo asesor, pero cuanto más cercano se esté a poder plantear cuestiones decisivas para la agricultura familiar, el valor de la tarea del Consejo será mayor" (CANPO). En el mismo sentido hubo referencias a su jerarquía: "creo que tiene que estar mucho más jerarquizada la presencia ahí en el Consejo, el Consejo debería estar más jerarquizado" (AGA). A su vez las menciones también fueron en la línea de proponer posibles acciones del Consejo, "que tome el lugar que debe tomar, que debe ser con mucho más protagonismo y participación de las organizaciones para que las instituciones puedan tomar nota y a partir de eso implementar las políticas" ( $\mathrm{MNCl}$-Somos Tierra) y la necesidad de "fortalecerlo y hacer del Consejo 
de la agricultura familiar un lugar de debate de política pública y de ejecución de esa política pública" (FECOFE).

Por el lado de las consideraciones acerca de roles no establecidos en la normativa se expresó la necesidad de que el Consejo tome la tarea de "presentar proyectos alternativos a los distintos proyectos hegemónicos que van queriendo caminar en el país como son los inteligenciados desde el consejo agroexportador y agroindustrial" (MCL) y de "oponerse a algunas políticas hegemónicas y de concentración y de ofrecer al mismo tiempo propuestas alternativas" (MCL). Y en la misma línea de ampliar la mirada del Consejo se consideró que debe tomar su rol como un espacio para "debatir lo agrario en Argentina. Ya no solamente lo sectorial. Sino lo agrario. (...) No restringir el diálogo con el Estado a lo productivo y comercial, sino a todo lo que tiene que ver con el desarrollo y lo agrario dentro de esos modelos de desarrollo" (ACINA).

Estas preferencias acerca de lo que se espera del Consejo permiten establecer los niveles de participación que podría tener el espacio. Específicamente las propuestas apuntan a una participación interactiva (nivel seis) en la que las organizaciones puedan participar con mayores grados de control sobre los temas de discusión y una participación de nivel siete (auto-desarrollo) en la que el espacio tenga la iniciativa para discutir problemáticas más allá de las planteadas por los instrumentos normativos que lo estructuran.

\section{Conclusiones}

En el desarrollo de este trabajo se pudo ver cómo el proceso de institucionalización de la agricultura familiar, campesina e indígena en Argentina generó el contexto adecuado para la creación de un espacio participativo de decisión de políticas públicas: el Consejo de la Agricultura Familiar, Campesina e Indígena.

El espacio del Consejo tiene como finalidad expresa en la normativa que lo crea la de ser un espacio participativo para debatir y generar consensos sobre diseños e implementación de políticas públicas estratégicas para el desarrollo sustentable y consolidación de la Agricultura Familiar, Campesina e Indígena. Además por la ley 27.118 de agricultura familiar, campesina e indígena que lo recepta se le da participación en la aplicación de la misma.

Cuando comparamos estos objetivos con los beneficios considerados por parte de los y las representantes de las organizaciones que integran el Consejo vemos que su constitución como un espacio participativo y de articulación institucional se encuentra lograda en tanto en las respuestas siempre estuvo presente como un aspecto beneficioso el diálogo posible con el Estado y entre las mismas organizaciones, tanto para temas generales como para procesos específicos (como la sanción y reglamentación de la ley 27.118). 
Por otro lado observamos que los beneficios para las organizaciones y para la agricultura familiar, campesina e indígena no se agotan en los espacios de diálogo sino que los trascienden en tanto el Consejo representa una jerarquización y puesta en valor tanto de cada una de las organizaciones, como de la heterogeneidad que significan a nivel territorial y de la agricultura familiar, campesina e indígena en su conjunto. Esta finalidad no declarada se resignifica cuando se tiene en consideración la importancia de construir una agenda pública para los y las integrantes de la agricultura familiar, campesina e indígena.

Como una suerte de contraste con estos beneficios aparecen las expresiones a favor de mejorar el funcionamiento del Consejo (en tanto aumentar su protagonismo y su incidencia en la toma de decisiones, superando su rol de "consejo asesor") y la propuesta de que sea en el ámbito del Consejo donde se encabece la propuesta de modelos alternativos de desarrollo agrario y la oposición al modelo dominante.

Este trabajo tiene carácter exploratorio por lo que más que conclusiones lo que se propone son ideas sobre las cuáles seguir reflexionando para profundizar en el entendimiento del funcionamiento de los espacios participativos de decisión de políticas públicas. La primer idea que ofrecemos luego de realizado este trabajo es acerca de los límites del diseño institucional del espacio. Una estructura creada por resolución (una normativa que puede ser modificada o derogada sin pasar por ninguna instancia de discusión pública) y sin ningún mecanismo institucional de incidencia en la toma de decisiones (un derecho a veto, una opinión vinculante, un procedimiento estructurado de consideración de las propuestas del Consejo) se ve muy limitada en cuanto a la operativización de la mirada heterogénea y federal de las organizaciones sobre las políticas públicas que se discuten.

Y como segunda idea planteamos que existe un amplio horizonte de posibilidades para el Consejo en cuanto a su rol en el diseño e implementación de políticas públicas. Un horizonte que comienza en las propuestas emanadas de los y las protagonistas mismos del Consejo y que van en la línea de disputar los sentidos que las democracias representativas liberales le asignan a la idea de participación. De volver a pensar en una democracia como una "práctica política capaz de transformar las relaciones económicas y sociales, la distribución de roles, el hecho permanente" (Scavino, 1999, p. 51-52). Una democracia participativa y de alta intensidad. 


\section{Referencias bibliográficas}

Ambort, M. E. (2017). Procesos asociativos en la agricultura familiar: un análisis de las condiciones que dieron lugar al surgimiento y consolidación de organizaciones en el cinturón hortícola platense 2005 - 2015. UNLP. http://www.memoria.fahce.unlp.edu.ar/tesis/te.1441/te.1441.pdf

De Sousa Santos, B. (2006). Renovar la teoría crítica y reinventar la emancipación social (encuentros en Buenos Aires). CLACSO.

Fernández, L. (2018). Políticas públicas para la agricultura familiar en Argentina durante el período 1990-2015. Nuevos y conocidos elementos en la agenda de debate. Trabajo y sociedad, (30), 219-241. http://pert-uba.com.ar/ archivos/publicaciones/30_FERNANDEZ_LISANDRO_Agricultura_familiar.pdf

Foro Nacional de la Agricultura Familiar (2006). Lineamientos generales de políticas públicas orientadas a la elaboración de un plan estratégico para la agricultura familiar. FAA- DDR.

Geilfus, F. (2002). 80 herramientas para el desarrollo participativo: diagnóstico, planificación, monitoreo, evaluación. IICA.

Gisclard, M., Allaire, G., \& Cittadini, R. (2015). Proceso de institucionalización de la agricultura familiar y nuevo referencial para el desarrollo rural en la Argentina. Mundo Agrario, 16(31), 1-18. http://www.scielo.org.ar/pdf/magr/v16n31/v16n31a03.pdf

Gonzalez, E., Gómez, C., \& Moricz, M. (2013). Normativas vinculadas a los procesos de producción y comercialización de la agricultura familiar urbana y periurbana. INTA.

Hang, G. M., Ferraris, G., Larrañaga, G., Seibane, C., \& Kebat, C. (2015). El contexto, las políticas públicas y su relación con la horticultura en La Plata, Argentina. Revista Facultad de Agronomía, 114(3) 222-231.

http://revista.agro.unlp.edu.ar/index.php/revagro/article/view/358

INTA. (2005). Programa Nacional de Investigación y Desarrollo Tecnológico para la Pequeña Agricultura Familiar (PNPAF). Documento base. INTA.

Marradi, A., Archenti, N., \& Piovani, J. I. (2007). Metodología de las ciencias sociales. Emecé.

Scavino, D. (1999). La era de la desolación. Manantial.

Scharpf, F. W. (1997). Games Real Actors Play: Actor-Centred Institutionalism in Policy Research. Westview Press.

Schiavoni, G. (2010). Describir y prescribir: la tipificación de la agricultura familiar en la Argentina. En M. Manzanal \& G. Neiman, Las agriculturas familiares del MERCOSUR. Trayectorias, amenazas y desafíos (pp. 43- 59). Ciccus. 


\section{Agradecimientos}

Agradecemos la buena predisposición de los y las representantes de las organizaciones de la agricultura familiar, campesina e indígena que con su participación hicieron posible este trabajo: Diego Montón (MNCl-Somos Tierra), Eduardo Sorazabal (AGA), Hipólito Madariaga (Frente Agrario Evita), José Luis Livolti (MCL), Juan Carlos "Tato" Figueredo (ACINA), Juan Manuel Rossi (FECOFE), Mónica Polidoro (AMRAF), Pedro Oroquieta (UAPA), Roberto Solano (FNC) y Sergio Dumrauf (CANPO).

Dirección de correspondencia:

Rodrigo Palleres Balboa

Contacto: rodrigo_palleres@hotmail.com 
Los organismos participativos para políticas públicas de la agricultura familiar, campesina e indígena por Rodrigo Palleres Balboa, Edgardo Gabriel González y Guillermo Miguel Hang

\section{ANEXO - Organizaciones de la agricultura familiar, campesina, indígena que integran el Consejo de la Agricultura Familiar, Campesina, Indígena.}

\begin{tabular}{|c|c|c|c|c|}
\hline Organización & $\begin{array}{l}\text { Presencia territorial } \\
\text { predominante }^{12}\end{array}$ & $\begin{array}{l}\text { Tipos de producción } \\
\text { predominantes }\end{array}$ & $\begin{array}{l}\text { Personería } \\
\text { jurídica }\end{array}$ & $\begin{array}{c}\text { Participación en el } \\
\text { Consejo }\end{array}$ \\
\hline $\begin{array}{l}\text { Agrupación Grito } \\
\text { de Alcorta (AGA) }\end{array}$ & $\begin{array}{l}\text { Región Pampeana y } \\
\text { Noroeste }\end{array}$ & Agrícola-ganadera & No & $\begin{array}{l}\text { Desde el comienzo } \\
\text { ininterrumpidamente }\end{array}$ \\
\hline $\begin{array}{l}\text { Asamblea } \\
\text { Campesina e } \\
\text { Indígena del } \\
\text { Norte Argentino } \\
\text { (ACINA) }\end{array}$ & Región Noroeste & $\begin{array}{l}\text { Apícola, ganadera, } \\
\text { frutihortícola, caprina, } \\
\text { cabriteros entre otras }\end{array}$ & No & $\begin{array}{l}\text { Desde el comienzo } \\
\text { ininterrumpidamente }\end{array}$ \\
\hline $\begin{array}{l}\text { Asociación de } \\
\text { Mujeres Rurales } \\
\text { Argentinas } \\
\text { (AMRAF) }\end{array}$ & $\begin{array}{l}\text { Región Pampeana, } \\
\text { Noroeste y Noreste }\end{array}$ & $\begin{array}{l}\text { Agrícola-ganadera, } \\
\text { hortícola, agregado de } \\
\text { valor }\end{array}$ & $\mathrm{Si}$ & $\begin{array}{l}\text { Desde el comienzo } \\
\text { ininterrumpidamente }\end{array}$ \\
\hline $\begin{array}{l}\text { Corriente } \\
\text { Agraria Nacional } \\
\text { y Popular } \\
\text { (CANPO) }\end{array}$ & $\begin{array}{l}\text { Región Pampeana, } \\
\text { Noroeste, Noreste } \\
\text { y Cuyo }\end{array}$ & $\begin{array}{l}\text { Agrícola-ganadera, } \\
\text { hortícola y vitivinícola }\end{array}$ & No & Desde el año 2020 \\
\hline $\begin{array}{l}\text { Encuentro } \\
\text { Nacional de } \\
\text { Organizaciones } \\
\text { Territoriales } \\
\text { de Pueblos } \\
\text { Originarios } \\
\text { (ENOTPO) }\end{array}$ & $\begin{array}{l}\text { Región Noroeste, } \\
\text { Noreste, Cuyo y } \\
\text { Patagonia }\end{array}$ & $\begin{array}{l}\text { Ganadería a baja escala, } \\
\text { agricultura, producción } \\
\text { artesanal, turismo, cría } \\
\text { de animales chicos, } \\
\text { corte de junquillo, } \\
\text { apicultura, aves de } \\
\text { corral y frutihortícola }\end{array}$ & No & $\begin{array}{l}\text { Desde el comienzo } \\
\text { ininterrumpidamente }\end{array}$ \\
\hline $\begin{array}{l}\text { Federación } \\
\text { Agraria } \\
\text { Argentina (FAA) }\end{array}$ & $\begin{array}{l}\text { Región Pampeana, } \\
\text { Cuyo y Noroeste }\end{array}$ & $\begin{array}{l}\text { Agrícola-ganadera, } \\
\text { porcina, algodonera, } \\
\text { tambera }\end{array}$ & $\mathrm{Si}$ & $\begin{array}{l}\text { Desde el comienzo } \\
\text { interrumpidamente }\end{array}$ \\
\hline $\begin{array}{l}\text { Federación de } \\
\text { Cooperativas } \\
\text { Federadas } \\
\text { (FECOFE) }\end{array}$ & $\begin{array}{l}\text { Región Pampeana, } \\
\text { Noroeste y Cuyo }\end{array}$ & $\begin{array}{l}\text { Agrícola-ganadera, } \\
\text { vitivinícola, cítricos y } \\
\text { tambo }\end{array}$ & $\mathrm{Si}$ & Desde el año 2020 \\
\hline $\begin{array}{l}\text { Federación de } \\
\text { Organizaciones } \\
\text { Nucleadas de } \\
\text { la Agricultura } \\
\text { Familiar (FONAF) }\end{array}$ & $\begin{array}{l}\text { Región Pampeana, } \\
\text { Cuyo, Patagonia y } \\
\text { Noreste }\end{array}$ & $\begin{array}{l}\text { Agrícola-ganadera, } \\
\text { vitivinícola, } \\
\text { frutihortícola, pesca } \\
\text { artesanal, entre otras }\end{array}$ & $\mathrm{Si}$ & $\begin{array}{l}\text { Desde el comienzo } \\
\text { ininterrumpidamente }\end{array}$ \\
\hline $\begin{array}{l}\text { Federación } \\
\text { Nacional } \\
\text { Campesina } \\
\text { (FNC) }\end{array}$ & $\begin{array}{l}\text { Región Pampeana, } \\
\text { Noreste y Noroeste }\end{array}$ & $\begin{array}{l}\text { Hortícola, florícola, } \\
\text { agrícola-ganadera }\end{array}$ & No & Desde el año 2020 \\
\hline
\end{tabular}

12 Tomamos las macro regiones Región Pampeana, Noroeste, Noreste, Patagonia y Cuyo. 
Pensamiento y Acción Interdisciplinaria, Volumen 7, número 1, año 2021. ISSN 0719-8078. pp. 129-145

\begin{tabular}{|c|c|c|c|c|}
\hline $\begin{array}{l}\text { Frente Agrario } \\
\text { Evita }\end{array}$ & $\begin{array}{l}\text { Región Pampeana, } \\
\text { Noreste y Noroeste }\end{array}$ & $\begin{array}{l}\text { Hortícola, florícola y } \\
\text { apícola }\end{array}$ & No & $\begin{array}{l}\text { Desde el comienzo } \\
\text { ininterrumpidamente }\end{array}$ \\
\hline $\begin{array}{l}\text { Frente Nacional } \\
\text { Campesino }\end{array}$ & Región Noreste & $\begin{array}{l}\text { Aprovechamiento } \\
\text { de bosque nativo, } \\
\text { ganadería, apícola entre } \\
\text { otras }\end{array}$ & No & $\begin{array}{l}\text { Desde el comienzo } \\
\text { ininterrumpidamente }\end{array}$ \\
\hline $\begin{array}{l}\text { La Campora } \\
\text { Rural }\end{array}$ & Región Pampeana & Agrícola-ganadera & No & $\begin{array}{l}\text { Desde el año } 2015 \\
\text { interrumpidamente }\end{array}$ \\
\hline $\begin{array}{l}\text { Movimiento } \\
\text { Agroecológico } \\
\text { de América } \\
\text { Latina y el Caribe } \\
\text { (MAELA) }\end{array}$ & $\begin{array}{l}\text { Región Noreste, } \\
\text { Noroeste y } \\
\text { Pampeana }\end{array}$ & $\begin{array}{l}\text { Hortícola, } \\
\text { cunícola, avícola y } \\
\text { agrícola-ganadera }\end{array}$ & $\mathrm{Si}$ & $\begin{array}{l}\text { Desde el comienzo } \\
\text { interrumpidamente }\end{array}$ \\
\hline $\begin{array}{l}\text { Movimiento } \\
\text { Campesino de } \\
\text { Liberación }\end{array}$ & $\begin{array}{l}\text { Región Noreste, } \\
\text { Noroeste y } \\
\text { Pampeana }\end{array}$ & $\begin{array}{l}\text { Agrícola-ganadera, } \\
\text { porcina y caprina }\end{array}$ & No & $\begin{array}{l}\text { Desde el comienzo } \\
\text { ininterrumpidamente }\end{array}$ \\
\hline $\begin{array}{l}\text { Movimiento de } \\
\text { Trabajadores } \\
\text { Excluidos - } \\
\text { Rama Rural }\end{array}$ & $\begin{array}{l}\text { Región Pampeana y } \\
\text { Patagonia }\end{array}$ & $\begin{array}{l}\text { Hortícola, florícola, } \\
\text { cebollera, } \\
\text { apícola, caprina y } \\
\text { agrícola-ganadera }\end{array}$ & No & Desde el año 2020 \\
\hline $\begin{array}{l}\text { Organización } \\
\text { Nacional } \\
\text { de Pueblos } \\
\text { Indígenas en } \\
\text { Argentina } \\
\text { (ONPIA) }\end{array}$ & $\begin{array}{l}\text { Región Patagonia, } \\
\text { Noroeste y Noreste }\end{array}$ & $\begin{array}{l}\text { Agricultura, cría de } \\
\text { animales chicos, } \\
\text { apicultura, aves de } \\
\text { corral y frutihortícola } \\
\text { entre otras }\end{array}$ & $\mathrm{Si}$ & $\begin{array}{l}\text { Desde el comienzo } \\
\text { ininterrumpidamente }\end{array}$ \\
\hline $\begin{array}{l}\text { Unión Argentina } \\
\text { de Pescadores } \\
\text { Artesanales } \\
\text { (UAPA) }\end{array}$ & $\begin{array}{l}\text { Patagonia y Región } \\
\text { Pampeana }\end{array}$ & $\begin{array}{l}\text { Pesca artesanal de mar } \\
\text { y río }\end{array}$ & $\mathrm{Si}$ & $\begin{array}{l}\text { Desde el comienzo } \\
\text { ininterrumpidamente }\end{array}$ \\
\hline
\end{tabular}

Fuente: elaboración propia en base a la información proporcionada por informantes calificados y las entrevistas estructuradas realizadas en el marco de esta investigación. 